\title{
Novel composite platform: Biomolecules-incorporation for biocatalysis, separation and biopharmaceutical formulations
}

\author{
Yao Chen \\ Nankai University, Tianjin, China, People's Republic of; \\ chenyao@nankai.edu.cn
}

Biomacromolecules, such as enzymes, are ubiquitous in nature and essential for maintaining basic life activities. Apart from the fundamental biological functions, biomacromolecules are also of great values in industrial applications, especially in food and pharmaceutical production. However, their industrial applications are often handicapped by low operational stability, poor robustness, difficult recovery and reuse. Incorporation of biomolecules within protective exteriors has been proved to be an effective method to promote their stabilities and applications. As new classes of crystalline solid-state materials, porous frameworks materials (such as covalent-organic frameworks, COFs and metal-organic frameworks, MOFs) feature high surface area, tunable pore size, high stability, and easily tailored functionality, which entitle them as ideal supports for encapsulation of biomolecules to form novel composite materials for various applications. Moreover, the formed composites can combine the properties of both constitutes, where crystalline frameworks materials and biomolecules are indeed mutually beneficial. Our researches mainly focus on their biocatalysis, separation and medicinal applications. This novel crystalline platform composed of biomolecules-incorporation and framework materials exhibited various functionality and superior poteintials in biocatalysis, bioseparation, and biopharmaceutical formulations.

Keywords: Biomolecules; biocatalysis; separation; biopharmaceutical 